九州大学学術情報リポジトリ

Kyushu University Institutional Repository

\title{
On the global uniqueness of Stokes' wave of extreme form
}

Kobayashi, Kenta

Faculty of Mathematics, Kyushu University

http://hdl. handle. net/2324/4022

出版情報：MHF Preprint Series. 2007-8，2007-02-14. 九州大学大学院数理学研究院 バージョン：

権利関係 : 


\title{
MHF Preprint Series
}

Kyushu University

21st Century COE Program

Development of Dynamic Mathematics with

High Functionality

\section{On the global uniqueness of Stokes' wave of extreme form}

\author{
K. Kobayashi
}

MHF 2007-8

( Received February 14, 2007 )

Faculty of Mathematics

Kyushu University

Fukuoka, JAPAN 


\title{
On the global uniqueness of Stokes' wave of extreme form \\ Kenta Kobayashi 1
}

\begin{abstract}
We present a computer-assisted proof of global uniqueness of Stokes' wave of extreme form. Stokes' wave of extreme form is a water wave which forms a corner of 120 degrees at the crest, and is considered to be the limit of the positive solution of Nekrasov's equation which expresses periodic gravity waves of permanent form on the free surface. The numerical verification method plays an important role in the proof. As for the global uniqueness of Stokes' wave of extreme form, it is not only a longtime open problem, but also it is related to an important conjecture which is called Stokes' conjecture.
\end{abstract}

Key Words: Stokes' wave of extreme form, Nekrasov's equation, Numerical verification method, gravity wave, Stokes' conjecture

\section{Introduction}

We are concerned in this paper with Stokes' wave of extreme form (see, e.g., [8]), which is a positive solution of a nonlinear integral equation for the unknown $\theta:(0, \pi] \rightarrow \mathbb{R}$ and is written as follows:

$$
\left\{\begin{array}{l}
\theta(s)=\int_{0}^{\pi} K(s, t) \frac{\sin \theta(t)}{\int_{0}^{t} \sin \theta(w) d w} d t \\
0<\theta(s)<\frac{\pi}{2} \quad s \in(0, \pi), \\
\theta(\pi)=0,
\end{array}\right.
$$

where

$$
K(s, t)=\frac{2}{3 \pi} \sum_{k=0}^{\infty} \frac{\sin k s \sin k t}{k}=\frac{1}{3 \pi} \log \left|\frac{\sin \frac{s+t}{2}}{\sin \frac{s-t}{2}}\right| .
$$

Stokes' wave of extreme form is derived from the following equation by Nekrasov:

$$
\left\{\begin{array}{l}
\theta(s)=\int_{0}^{\pi} K(s, t) \frac{\sin \theta(t)}{\mu^{-1}+\int_{0}^{t} \sin \theta(w) d w} d t \\
0<\theta(s)<\frac{\pi}{2} \quad s \in(0, \pi), \\
\theta(0)=\theta(\pi)=0,
\end{array}\right.
$$

where $\mu$ is a positive parameter. The equation (1.1) is derived from this by letting $\mu \rightarrow \infty$.

Nekrasov's equation arises from the following physical situation. We consider a twodimensional, irrotational motion of inviscid fluid having a free surface. In a coordinate

\footnotetext{
${ }^{1}$ Faculty of Mathematics, Kyushu University, Fukuoka 812-8581 Japan.

This research is partly supported by the Grant-in-Aid for JSPS Fellows, The Ministry of Education, Culture, Sports, Science and Technology, Japan and the 21st Century COE program of Faculty of Mathematics, Kyushu University
} 
system moving with the wave, the wave profile is assumed to be stationary. We further assume that the shape is periodic and that the flow is infinitely deep. We are then asked to determine the shape of the free surface. Details are omitted (see [8]) but we should note that the free surface is represented as $(x(s), y(s)) \quad(0<s<2 \pi)$, where $x$ and $y$ are determined by

$$
\frac{d x}{d s}=-\frac{L}{2 \pi} e^{-H \theta(s)} \cos \theta(s), \quad \frac{d y}{d s}=-\frac{L}{2 \pi} e^{-H \theta(s)} \sin \theta(s) .
$$

Here, $L$ is the wavelength and $H$ is the Hilbert transform. The right hand sides are known if $\theta$ is. Thus, after integrating in $s$, we have a parametric representation of the free surface.

The wave is assumed (i) to be symmetric about its crest, (ii) to have a surface that is a single-valued $2 \pi$-periodic function of horizontal distance, and (iii) to have only one peak and one trough per period. As a consequence we have

$$
\theta(s)=-\theta(-s), \quad \theta(s+2 \pi)=\theta(s), \quad \text { and } \quad 0<\theta(s)<\frac{\pi}{2} \text { on }(0, \pi) .
$$

We call such solutions positive solutions.

We finally note that

$$
\mu=\frac{3 g L}{2 \pi c^{2}} e^{-3 H \theta(0)}
$$

where $c$ denotes the propagation speed, and $g$ the gravitational acceleration. We refer the reader to [8] for the derivation of (1.2) and (1.3).

The global nature of the positive solutions for (1.2) was shown first by Krasovskii[5]. He showed that, for any $\beta \in(0, \pi / 6)$, the equation (1.2) has a solution $(\mu, \theta)$ satisfying (1.4) and $\max \theta(s)=\beta$. Presumably Krasovskii imagined that his solutions formed the whole branch up to the extreme wave. However, the fact of the matter was not that simple: numerical experiments showed strong evidence, that there existed solutions in which the maximum slope was strictly bigger than $\pi / 6$ (see [10]). The existence of a positive solution for every $\mu>3$ (there is no positive solution when $\mu \leq 3$ ) was first proved by Keady \& Norbury[4].

When $\mu \rightarrow \infty$, the speed at the crest tends to zero and the crest becomes sharper. Keady and Norbury guaranteed all waves having one crest and one trough except for the case of $\mu=\infty$. The waves of extreme form satisfies (1.1). The existence of a solution to (1.1) was proved by Toland[12]. He proved that as $\mu \rightarrow \infty$, the solution of (1.2) have a convergent subsequence and that the limit function satisfies (1.1).

About the extreme wave, Stokes[11] recognized the following two propositions which are nowadays called Stokes' conjectures:

(a) the crest forms a corner of angle $2 \pi / 3$, that is, $\lim _{s \downarrow 0} \theta(s)=\pi / 6$.

(b) the wave profile between two consecutive crests is concave, that is, $\theta^{\prime}(s)<0$ for $s \in(0, \pi)$.

The conjecture (a) was proved by Amick et al.[1]. Toland \& Plotnikov[13] proved that there exists a wave which satisfies $\theta^{\prime}(s)<0$. However, this does not settle the conjecture (b) completely. Because of the lack of the proof of the uniqueness, it is not excluded that there is a positive solution of (1.1) which does not satisfy $\theta^{\prime}(s)<0$. 
Although the existence of solutions for Nekrasov's equation and Stokes' wave of extreme form are proved, despite the effort of many mathematicians for many decades, the global uniqueness seems to remain open. While writing this paper, the author heard that Fraenkel[2] proved the local uniqueness, i.e., the uniqueness in a certain neighborhood of the solution. On the other hand, we had succeeded to prove the global uniqueness of a positive solution for Nekrasov's equation when $\mu \leq 170$ (see [3]). With the technique in [3], we present in this paper a proof of the global uniqueness of Stokes' wave of extreme form. In the two proofs, we employ the numerical verification method (see, for instance, [9], [6]) to obtain rigorous mathematical results by numerical computations.

The present paper is organized as follows: In section 2, we explain the idea of the proof of uniqueness. Discretization is explained in section 3, and we show the numerical results in section 4 .

Influence of discretization in section 3 and rounding error in section 4 are both rigorously evaluated. Consequently, though we use numerical computation, our result is rigorous.

In this paper, $\theta$ always denotes Stokes' wave of extreme form, namely, a solution of (1.1), $s$ is assumed to run in $0<s \leq \pi$. $1_{A}$ denotes the function which takes value 1 if condition $A$ holds, and takes value 0 otherwise.

\section{The idea of the proof of uniqueness}

In this section, we first derive a formula about bounds of $\theta$. Secondly, we present the specific lower bound of $\theta$. Finally, we explain how to prove the uniqueness.

First of all, we prove the following theorem,

Theorem 2.1 Assume that, for $\theta(s)$, an upper bound and a lower bound are given as

$$
0 \leq \underline{\theta}(s) \leq \theta(s) \leq \bar{\theta}(s) \leq \pi / 2,
$$

then, it holds that

$$
J(\underline{\theta}, \bar{\theta})(s) \leq \theta(s) \leq J(\bar{\theta}, \underline{\theta})(s),
$$

where

$J(\phi, \varphi)(s)=\frac{1}{6 \pi} \int_{0}^{\pi} \cot \frac{t}{2} \log \left(1+\frac{\int_{|s-t|}^{\pi-|s+t-\pi|} \sin \phi(w) d w}{\int_{0}^{\min (s,|s-t|)} \sin \varphi(w) d w+\int_{\min (s,|s-t|)}^{|s-t|} \sin \phi(w) d w}\right) d t$.

Proof. Note first that

$$
\frac{\partial}{\partial t} K(s, t)=\frac{1}{6 \pi}\left(\cot \frac{t+s}{2}-\cot \frac{t-s}{2}\right)=\frac{1}{3 \pi} \cdot \frac{\sin s}{\cos t-\cos s} .
$$


With this and (1.1) we compute as follows.

$$
\begin{aligned}
& \theta(s)=\int_{0}^{\pi} K(s, t) \frac{d}{d t} \log \left(\int_{0}^{t} \sin \theta(w) d w\right) d t \\
& =\frac{1}{3 \pi} \int_{0}^{\pi} \frac{\sin s}{\cos s-\cos t} \cdot \log \left(\frac{\int_{0}^{t} \sin \theta(w) d w}{\int_{0}^{s} \sin \theta(w) d w}\right) d t \\
& \leq \frac{1}{3 \pi} \int_{0}^{\pi} \frac{\sin s}{\cos s-\cos t} \cdot \log \left(\frac{\int_{0}^{t} \sin \left(1_{w<s} \cdot \theta(w)+1_{w \geq s} \cdot \bar{\theta}(w)\right) d w}{\int_{0}^{s} \sin \theta(w) d w}\right) d t \\
& =\frac{1}{6 \pi} \int_{0}^{\pi}\left(\cot \frac{t-s}{2}-\cot \frac{t+s}{2}\right) \log \left(\frac{\int_{0}^{t} \sin \left(1_{w<s} \cdot \theta(w)+1_{w \geq s} \cdot \bar{\theta}(w)\right) d w}{\int_{0}^{s} \sin \theta(w) d w}\right) d t \\
& =\frac{1}{6 \pi} \int_{-\pi}^{\pi} \cot \frac{t-s}{2} \cdot \log \left(\frac{\int_{0}^{|t|} \sin \left(1_{w<s} \cdot \theta(w)+1_{w \geq s} \cdot \bar{\theta}(w)\right) d w}{\int_{0}^{s} \sin \theta(w) d w}\right) d t \\
& =\frac{1}{6 \pi} \int_{0}^{\pi} \cot \frac{t}{2} \cdot \log \left(\frac{\int_{0}^{\pi-|s+t-\pi|} \sin \left(1_{w<s} \cdot \theta(w)+1_{w \geq s} \cdot \bar{\theta}(w)\right) d w}{\int_{0}^{s} \sin \theta(w) d w}\right) d t \\
& +\frac{1}{6 \pi} \int_{-\pi}^{0} \cot \frac{t}{2} \cdot \log \left(\frac{\int_{0}^{|s+t|} \sin \left(1_{w<s} \cdot \theta(w)+1_{w \geq s} \cdot \bar{\theta}(w)\right) d w}{\int_{0}^{s} \sin \theta(w) d w}\right) d t \\
& =\frac{1}{6 \pi} \int_{0}^{\pi} \cot \frac{t}{2} \cdot \log \left(\frac{\int_{0}^{\pi-|s+t-\pi|} \sin \left(1_{w<s} \cdot \theta(w)+1_{w \geq s} \cdot \bar{\theta}(w)\right) d w}{\int_{0}^{|s-t|} \sin \left(1_{w<s} \cdot \theta(w)+1_{w \geq s} \cdot \bar{\theta}(w)\right) d w}\right) d t \\
& =\frac{1}{6 \pi} \int_{0}^{\pi} \cot \frac{t}{2} \cdot \log \left(1+\frac{\int_{|s-t|}^{\pi-|s+t-\pi|} \sin \left(1_{w<s} \cdot \theta(w)+1_{w \geq s} \cdot \bar{\theta}(w)\right) d w}{\int_{0}^{\min (s,|s-t|)} \sin \theta(w) d w+\int_{\min (s,|s-t|)}^{|s-t|} \sin \bar{\theta}(w) d w}\right) d t \\
& \leq \frac{1}{6 \pi} \int_{0}^{\pi} \cot \frac{t}{2} \cdot \log \left(1+\frac{\int_{|s-t|}^{\pi-|s+t-\pi|} \sin \bar{\theta}(w) d w}{\int_{0}^{\min (s,|s-t|)} \sin \underline{\theta}(w) d w+\int_{\min (s,|s-t|)}^{|s-t|} \sin \bar{\theta}(w) d w}\right) d t .
\end{aligned}
$$

Hence, $J(\bar{\theta}, \underline{\theta})(s)$ is a renewed upper bound of $\theta(s)$. In the same way, $J(\underline{\theta}, \bar{\theta})(s)$ is shown to be a renewed lower bound of $\theta(s)$.

This theorem is used in section 4 to obtain sharp upper and lower bounds of $\theta(s)$ by iteration. 
Next, we prove the following theorem about the specific lower bound of $\theta(s)$.

\section{Theorem 2.2}

$$
\theta(s) \geq 0.00005 \cdot 1_{0<s \leq \pi / 2}
$$

Proof. Let $\varepsilon$ denote 0.00005 . Assume that $0<a<\pi / 2$ satisfies the following condition;

$$
\text { for all } 0<s \leq a, \quad \theta(s) \geq \varepsilon \text { holds. }
$$

Since Amick et al. [1] proved that $\lim _{s \downarrow 0} \theta(s)=\pi / 6$, such an ' $a$ ' certainly exists.

Then, from Theorem 2.1, for $a \leq s \leq \min (1.01 a, \pi / 2)$,

$$
\begin{aligned}
\theta(s) & \geq J\left(\varepsilon \cdot 1_{s \leq a}, \pi / 2\right)(s) \\
& \geq \frac{1}{6 \pi} \int_{s-a}^{s+a} \cot \frac{t}{2} \cdot \log \left(1+\frac{a-|s-t|}{|s-t|} \sin \varepsilon\right) d t \\
& =\frac{1}{3 \pi} \int_{0}^{a} \frac{\sin s}{\cos t-\cos s} \cdot \log \left(1+\frac{a-t}{t} \sin \varepsilon\right) d t \\
& \geq \frac{1}{3 \pi} \int_{0}^{a} \frac{1}{s-t} \cdot \log \left(1+\frac{a-t}{t} \sin \varepsilon\right) d t \\
& \geq \frac{1}{3 \pi} \cdot \frac{1}{s} \int_{0}^{a} \log \left(1+\frac{a-t}{t} \sin \varepsilon\right) d t \\
& =\frac{1}{3 \pi} \cdot \frac{a}{s} \cdot \frac{\sin \varepsilon}{1-\sin \varepsilon} \cdot \log \left(\frac{1}{\sin \varepsilon}\right) \\
& \geq \frac{1}{3 \pi} \cdot \frac{\sin \varepsilon}{1.01} \cdot \log \left(\frac{1}{\sin \varepsilon}\right)=0.0000520194 \cdots \geq \varepsilon .
\end{aligned}
$$

Therefore, if $\theta(s) \geq \varepsilon$ holds for $0<s \leq a$, then its also holds for $0<s \leq$ $\min (1.01 a, \pi / 2)$. Inductively, $\theta(s) \geq \varepsilon$ holds for all $0<s \leq \pi / 2$.

Now, we will explain how to prove the uniqueness of (1.1). For this purpose, we use the following theorem.

Theorem 2.3 The solution of (1.1) is globaly unique if the following condition holds,

$$
\sup _{0<s \leq \pi} \frac{G(\bar{\theta}, \underline{\theta}, g)(s)}{g(s)}<1,
$$

where $\underline{\theta}(s) \geq 0$ and $\bar{\theta}(s) \leq \pi / 2$ are lower and upper bound of the solution of (1.1), $g(s)$ is an arbitrary positive function, and

$$
\begin{aligned}
G(\phi, \varphi, g)(s)=\frac{1}{6 \pi} \int_{0}^{\pi} \cot & \frac{t}{2} \cdot\left(\frac{\int_{|s-t|}^{\pi-|s+t-\pi|} \sin \phi(w) d w \cdot \int_{0}^{\min (s,|s-t|)} \cos \varphi(w) \cdot g(w) d w}{\int_{0}^{|s-t|} \sin \varphi(w) d w}\right. \\
& \left.+\int_{|s-t|}^{\pi-|s+t-\pi|} \cos \varphi(w) \cdot g(w) d w\right) \cdot \frac{d t}{\int_{0}^{\pi-|s+t-\pi|} \sin \varphi(w) d w} .
\end{aligned}
$$


Proof. Suppose that $\bar{\theta}(s) \geq 0$ and $\underline{\theta}(s) \leq \pi / 2$ are given. We then define inductively the following functions:

$$
\left\{\begin{aligned}
\phi_{0}(s) & =\bar{\theta}(s), \quad \varphi_{0}(s)=\underline{\theta}(s), & & \\
\phi_{n+1}(s) & =\min \left(\phi_{n}(s), J\left(\phi_{n}, \varphi_{n}\right)(s)\right), & & (n=0,1, \cdots), \\
\varphi_{n+1}(s) & =\max \left(\varphi_{n}(s), J\left(\varphi_{n}, \phi_{n}\right)(s)\right), & & (n=0,1, \cdots) .
\end{aligned}\right.
$$

Theorem 2.1 shows that for all $n \geq 0,0 \leq \varphi_{n}(s) \leq \theta(s) \leq \phi_{n}(s) \leq \pi / 2$.

By these definitions,

$$
\begin{aligned}
& \frac{\phi_{n+1}(s)-\varphi_{n+1}(s)}{g(s)} \leq \frac{J\left(\phi_{n}, \varphi_{n}\right)(s)-J\left(\varphi_{n}, \phi_{n}\right)(s)}{g(s)} \\
& =\frac{1}{6 \pi g(s)} \int_{0}^{\pi} \cot \frac{t}{2} \cdot \log \left(\frac{1+\frac{\int_{|s-t|}^{\pi-|s+t-\pi|} \sin \phi_{n}(w) d w}{\int_{0}^{\min (s,|s-t|)} \sin \varphi_{n}(w) d w+\int_{\min (s,|s-t|)}^{|s-t|} \sin \phi_{n}(w) d w}}{1+\frac{\int_{|s-t|}^{\pi-|s+t-\pi|} \sin \varphi_{n}(w) d w}{\int_{0}^{\min (s,|s-t|)} \sin \phi_{n}(w) d w+\int_{\min (s,|s-t|)}^{|s-t|} \sin \varphi_{n}(w) d w}}\right) d t . \\
& =\frac{1}{6 \pi g(s)} \int_{0}^{\pi} \cot \frac{t}{2} \cdot \log \left[1+\left\{\frac{\int_{|s-t|}^{\pi-|s+t-\pi|} \sin \phi_{n}(w) d w}{\int_{0}^{\min (s,|s-t|)} \sin \varphi_{n}(w) d w+\int_{\min (s,|s-t|)}^{|s-t|} \sin \phi_{n}(w) d w}\right.\right. \\
& \times\left(\int_{0}^{\min (s,|s-t|)}\left(\sin \phi_{n}(w)-\sin \varphi_{n}(w)\right) d w-\int_{\min (s,|s-t|)}^{|s-t|}\left(\sin \phi_{n}(w)-\sin \varphi_{n}(w)\right) d w\right) \\
& \left.+\int_{|s-t|}^{\pi-|s+t-\pi|}\left(\sin \phi_{n}(w)-\sin \varphi_{n}(w)\right) d w\right\} \\
& \left.\times \frac{1}{\int_{0}^{\min (s,|s-t|)} \sin \phi_{n}(w) d w+\int_{\min (s,|s-t|)}^{\pi-s+t-\pi \mid} \sin \varphi_{n}(w) d w}\right] d t \\
& \leq \frac{1}{6 \pi g(s)} \int_{0}^{\pi} \cot \frac{t}{2} \cdot\left(\frac{\int_{|s-t|}^{\pi-|s+t-\pi|} \sin \phi_{n}(w) d w \cdot \int_{0}^{\min (s,|s-t|)}\left(\sin \phi_{n}(w)-\sin \varphi_{n}(w)\right) d w}{\int_{0}^{\min (s,|s-t|)} \sin \varphi_{n}(w) d w+\int_{\min (s,|s-t|)}^{|s-t|} \sin \phi_{n}(w) d w}\right. \\
& \left.+\int_{|s-t|}^{\pi-|s+t-\pi|}\left(\sin \phi_{n}(w)-\sin \varphi_{n}(w)\right) d w\right) \\
& \times \frac{d t}{\int_{0}^{\min (s,|s-t|)} \sin \phi_{n}(w) d w+\int_{\min (s,|s-t|)}^{\pi-|s+t-\pi|} \sin \varphi_{n}(w) d w}
\end{aligned}
$$




$$
\begin{aligned}
& \leq \frac{1}{6 \pi g(s)} \int_{0}^{\pi} \cot \frac{t}{2} \cdot\left(\frac{\int_{|s-t|}^{\pi-|s+t-\pi|} \sin \bar{\theta}(w) d w \cdot \int_{0}^{\min (s,|s-t|)} \cos \underline{\theta}(w) \cdot g(w) d w}{\int_{0}^{|s-t|} \sin \underline{\theta}(w) d w}\right. \\
& \left.\quad+\int_{|s-t|}^{\pi-|s+t-\pi|} \cos \underline{\theta}(w) \cdot g(w) d w\right) \cdot \frac{d t}{\int_{0}^{\pi-|s+t-\pi|} \sin \underline{\theta}(w) d w} \cdot \sup _{0 \leq s \leq \pi} \frac{\phi_{n}(s)-\varphi_{n}(s)}{g(s)} \\
& =\frac{G(\bar{\theta}, \underline{\theta}, g)(s)}{g(s)} \cdot \sup _{0<s \leq \pi} \frac{\phi_{n}(s)-\varphi_{n}(s)}{g(s)} .
\end{aligned}
$$

This leads us to

$$
\sup _{0<s \leq \pi} \frac{\phi_{n+1}(s)-\varphi_{n+1}(s)}{g(s)} \leq \sup _{0<s \leq \pi} \frac{G(\bar{\theta}, \underline{\theta}, g)(s)}{g(s)} \cdot \sup _{0<s \leq \pi} \frac{\phi_{n}(s)-\varphi_{n}(s)}{g(s)} .
$$

If the assumption of this theorem holds, then

$$
\sup _{0<s \leq \pi} \frac{\phi_{n}(s)-\varphi_{n}(s)}{g(s)} \rightarrow 0, \quad(n \rightarrow \infty),
$$

and this means that the solution of (1.1) is unique.

The next problem is to choose a suitable function $g(s)$. The simple choice $g(s) \equiv$ constant was found not to work. Our choice, which will be exhibited in section 4, is more involved.

\section{Discretization}

The main purpose of this section is to explain how to discretize functional $J(\cdot, \cdot)$ and $G(\cdot, \cdot, \cdot)$ by step functions.

Let $N$ be an arbitrary even number $\geq 4$, and let $Q_{N}$ denote the set of step functions which are constant in each intervals

$$
\frac{k \pi}{N}<s \leq \frac{(k+1) \pi}{N} \quad(k=0,1, \cdots, N-1) .
$$

Assume that $\underline{\theta}(s) \geq 0$ and $\bar{\theta}(s) \leq \pi / 2$ are lower and upper bounds of the solution $\theta(s)$, and that $g(s)$ is an arbitrary positive function. We also assume that $\underline{\theta}(s), \bar{\theta}(s)$ and $g(s)$ belong to $Q_{N}$.

We will constructively define step functions $\bar{J}_{*}, \underline{J}_{*}$ and $G_{*}$ in $Q_{N}$ which satisfy

$$
\begin{aligned}
\bar{J}_{*}(\bar{\theta}, \underline{\theta})(s) & \geq \theta(s), \\
\underline{J}_{*}(\underline{\theta}, \bar{\theta})(s) & \leq \theta(s), \\
G_{*}(\bar{\theta}, \underline{\theta}, g)(s) & \geq G(\bar{\theta}, \underline{\theta}, g)(s),
\end{aligned}
$$

as discretizations of $\bar{J}(\cdot, \cdot), \underline{J}(\cdot, \cdot)$ and $G(\cdot, \cdot, \cdot)$. 
The values of $\bar{J}_{*}, \underline{J}_{*}$ and $G_{*}$ in the subinterval (3.1) are denoted by $\bar{J}_{k}, \underline{J}_{k}$ and $G_{k}$. We may accordingly write as follows:

$$
\begin{gathered}
\bar{J}_{*}(\bar{\theta}, \underline{\theta})(s)=\sum_{k=0}^{N-1} 1_{\frac{k \pi}{N}<s \leq \frac{(k+1) \pi}{N}} \cdot \bar{J}_{k}(\bar{\theta}, \underline{\theta}), \\
\underline{J}_{*}(\underline{\theta}, \bar{\theta})(s)=\sum_{k=0}^{N-2} 1_{\frac{k \pi}{N}<s \leq \frac{(k+1) \pi}{N}} \cdot \underline{J}_{k}(\underline{\theta}, \bar{\theta}), \\
G_{*}(\bar{\theta}, \underline{\theta}, g)(s)=\sum_{k=0}^{N-1} 1_{\frac{k \pi}{N}<s \leq \frac{(k+1) \pi}{N}} \cdot G_{k}(\bar{\theta}, \underline{\theta}, g) .
\end{gathered}
$$

The precise definitions of $\bar{J}_{k}(\bar{\theta}, \underline{\theta})$ etc. are rather complicated and will be given later. To that end, we define the following symbols.

$$
\begin{aligned}
\bar{\Theta}(u) & \equiv \sup _{u<x \leq N} \sin \bar{\theta}\left(\frac{\pi}{N} x\right), \quad 0 \leq u<N, \\
\underline{\Theta}(u) & \equiv \inf _{0<x \leq \min (u, N)} \sin \underline{\theta}\left(\frac{\pi}{N} x\right), \quad 0<u, \\
\gamma(u) & \equiv \sup _{u<x \leq N} \cos \underline{\theta}\left(\frac{\pi}{N} x\right) \cdot g\left(\frac{\pi}{N} x\right), \quad 0 \leq u<N, \\
\bar{\Theta}(N) & \equiv \lim _{u \uparrow N} \bar{\Theta}(u), \quad \underline{\Theta}(0) \equiv \lim _{u \downarrow 0} \underline{\Theta}(u), \quad \gamma(N) \equiv \lim _{u \uparrow N} \gamma(u), \\
\bar{\Theta}_{a} & \equiv \bar{\Theta}(a), \quad \bar{\Psi}_{a}^{b} \equiv \int_{a}^{b} \bar{\Theta}(u) d u, \\
\underline{\Theta}_{a} & \equiv \underline{\Theta}(a), \quad \underline{\Psi}_{a}^{b} \equiv \int_{a}^{b} \underline{\Theta}(u) d u, \\
\gamma_{a} & \equiv \gamma(a), \quad \Gamma_{a}^{b} \equiv \int_{a}^{b} \gamma(u) d u, \\
c_{1} & \equiv \sup _{0<t \leq N} \frac{\bar{\Psi}_{0}^{t}}{\underline{\Psi}_{0}^{t}}, \quad c_{2} \equiv \sup _{0<t \leq N} \frac{\Gamma_{0}^{t}}{\underline{\Psi}_{0}^{t}}, \quad c_{3} \equiv \sup _{0<t \leq N} \frac{t}{\underline{\Psi}_{0}^{t}}, \\
f[w] & \equiv N-|w-N| .
\end{aligned}
$$

For each $s$ and integer $k$ such that

$$
\frac{k \pi}{N}<s \leq \frac{(k+1) \pi}{N} \quad(0 \leq k \leq N-1)
$$

the following inequality holds,

$$
\underline{\Theta}\left(\frac{(k+1) w}{s}\right) \leq \sin \theta(w) \leq \bar{\Theta}\left(\frac{k w}{s}\right) \quad(0<w \leq \pi) .
$$

In fact, note first that $\sin \theta(w) \leq \sin \bar{\theta}$. Since $\frac{k w}{s}<\frac{N w}{\pi}$, we have $\bar{\Theta}\left(\frac{k w}{s}\right) \geq \sin \bar{\theta}(w)$, which implies the inequality on the right hand side. The one on the left hand side is proved similarly. 
Using (3.5) and Theorem 2.1, we have

$$
\begin{aligned}
\theta(s) & \leq \frac{1}{6 \pi} \int_{0}^{\pi} \cot \frac{t}{2} \log \left(1+\frac{\int_{|s-t|}^{\pi-|s+t-\pi|} \bar{\Theta}\left(\frac{k w}{s}\right) d w}{\int_{0}^{\min (s,|s-t|)} \underline{\Theta}\left(\frac{(k+1) w}{s}\right) d w+\int_{\min (s,|s-t|)}^{|s-t|} \bar{\Theta}\left(\frac{k w}{s}\right) d w}\right) d t \\
& =\frac{1}{6 \pi} \int_{0}^{\frac{\pi}{s}} \frac{s}{\tan \frac{s t}{2}} \log \left(1+\frac{\int_{|t-1|}^{\frac{\pi}{s}-\left|1+t-\frac{\pi}{s}\right|} \bar{\Theta}(k w) d w}{\int_{0}^{\min (1,|t-1|)} \underline{\Theta}((k+1) w) d w+\int_{\min (1,|t-1|)}^{|t-1|} \bar{\Theta}(k w) d w}\right) d t \\
& \leq \frac{1}{6 \pi} \int_{0}^{\frac{N}{k}} \lim _{\sigma \downarrow \frac{k \pi}{N}} \frac{\sigma}{\tan \frac{\sigma t}{2}} \log \left(1+\frac{\int_{|t-1|}^{\frac{\pi}{\sigma}-\left|1+t-\frac{\pi}{\sigma}\right|} \bar{\Theta}(k w) d w}{\int_{0}^{\min (1,|t-1|)} \underline{\Theta}((k+1) w) d w+\int_{\min (1,|t-1|)}^{|t-1|} \bar{\Theta}(k w) d w}\right) d t \\
& \leq \sum_{l=0}^{N-1} \bar{A}_{k, l}(\bar{\theta}, \underline{\theta})+\sum_{l=k}^{N-1} \min \left(\bar{B}_{k, l}(\bar{\theta}, \underline{\theta}), \bar{C}_{k, l}(\bar{\theta}, \underline{\theta})\right) \equiv \bar{J}_{k}(\bar{\theta}, \underline{\theta}),
\end{aligned}
$$

where $\bar{A}_{k, l}, \bar{B}_{k, l}$ and $\bar{C}_{k, l}$ are defined as follows:

First, $\quad \bar{A}_{k, l}(\bar{\theta}, \underline{\theta}) \quad$ is defined for $l=0$ as

$$
\frac{1}{6 \pi} \int_{0}^{\frac{1}{N}} \frac{4 \bar{\Theta}_{k\left(1-\frac{1}{N}\right)}}{\frac{1}{k+1} \underline{\Psi}_{0}^{(k+1)\left(1-\frac{1}{N}\right)}+\left(\frac{1}{N}-t\right) \underline{\Theta}_{k+1}} d t
$$

for $k=0, \quad 1 \leq l$,

$$
\frac{1}{6 \pi} \int_{\frac{l}{N}}^{\frac{l+1}{N}} \frac{2 N}{l} \log \left(1+\frac{\frac{2 l}{N} \bar{\Theta}_{0}}{\underline{\Psi}_{0}^{\left(1-\frac{l+1}{N}\right)}+\left(\frac{l+1}{N}-t\right) \underline{\Theta}_{1-\frac{l}{N}}}\right) d t
$$

and for $1 \leq k, 1 \leq l$,

$$
\frac{1}{6 \pi} \int_{\frac{l}{N}}^{\frac{l+1}{N}} \frac{\frac{k \pi}{N}}{\tan \frac{k l \pi}{2 N^{2}}} \log \left(1+\frac{\min \left(\frac{1}{k} \bar{\Psi}_{k\left(1-\frac{l+1}{N}\right)}^{f\left[k\left(1+\frac{l+1}{N}\right)\right]}, \frac{2 l}{N} \bar{\Theta}_{k\left(1-\frac{l+1}{N}\right)}\right)}{\frac{1}{k+1} \underline{\Psi}_{0}^{(k+1)\left(1-\frac{l+1}{N}\right)}+\left(\frac{l+1}{N}-t\right) \underline{\Theta}_{(k+1)\left(1-\frac{l}{N}\right)}}\right) d t
$$


$\bar{B}_{k, l}(\bar{\theta}, \underline{\theta})$ is defined as $\infty$ if $l=0$;

for $k=0, \quad 1 \leq l \leq \frac{N}{2}-1$,

$$
\frac{1}{6 \pi} \int_{\frac{N}{l+1}}^{\frac{N}{l}} \frac{2(l+1)}{N} \log \left(1+\frac{2 \bar{\Theta}_{0}}{\underline{\Psi}_{0}^{1}+(t-2) \bar{\Theta}_{0}}\right) d t
$$

for $k=0, \quad \frac{N}{2} \leq l$,

$$
\frac{1}{6 \pi} \int_{\frac{N}{l+1}}^{\frac{N}{l}} \frac{2(l+1)}{N} \log \left(1+\frac{2 \bar{\Theta}_{0}}{\underline{\Psi}_{0}^{\frac{N}{l+1}-1}+\left(t-\frac{N}{l+1}\right) \underline{\Theta}_{\frac{N}{l}-1}}\right) d t
$$

for $k \geq 1, \quad 1 \leq l \leq \frac{N}{2}-1$,

$$
\frac{1}{6 \pi} \int_{\frac{N}{l+1}}^{\frac{N}{l}} \frac{\frac{k \pi}{N}}{\tan \frac{k \pi}{2(l+1)}} \log \left(1+\frac{\frac{1}{k} \bar{\Psi}_{k\left(\frac{N}{l+1}-1\right)}^{\left.f\left[\frac{N}{l+1}+1\right)\right]}}{\frac{1}{k+1} \underline{\Psi}_{0}^{k+1}+\frac{1}{k} \bar{\Psi}_{k}^{k\left(\frac{N}{l+1}-1\right)}+\left(t-\frac{N}{l+1}\right) \bar{\Theta}_{k\left(\frac{N}{l}-1\right)}}\right) d t
$$

and for $k \geq 1, \quad \frac{N}{2} \leq l$,

$$
\frac{1}{6 \pi} \int_{\frac{N}{l+1}}^{\frac{N}{l}} \frac{\frac{k \pi}{N}}{\tan \frac{k \pi}{2(l+1)}} \log \left(1+\frac{\frac{1}{k} \bar{\Psi}_{k\left(\frac{N}{l+1}-1\right)}^{f\left[k\left(\frac{N}{l+1}+1\right)\right]}}{\frac{1}{k+1} \underline{\Psi}_{0}^{(k+1)\left(\frac{N}{l+1}-1\right)}+\left(t-\frac{N}{l+1}\right) \underline{\Theta}_{(k+1)\left(\frac{N}{l}-1\right)}}\right) d t .
$$

$\bar{C}_{k, l}(\bar{\theta}, \underline{\theta})$ is defined as $\infty$ if $l=N-1$;

for $k=0, l \leq \frac{N}{2}-1$,

$$
\frac{1}{6 \pi} \int_{\frac{N}{l+1}}^{\frac{N}{l}} \frac{2}{t} \cdot \frac{2 \bar{\Theta}_{0}}{\underline{\Psi}_{0}^{1}+(t-2) \bar{\Theta}_{0}} d t ;
$$

for $k=0, \quad \frac{N}{2} \leq l \leq N-2$,

$$
\frac{1}{6 \pi} \int_{\frac{N}{l+1}}^{\frac{N}{l}} \frac{2}{t} \cdot \frac{2 \bar{\Theta}_{0}}{\underline{\Psi}_{0}^{\frac{N}{l+1}-1}+\left(t-\frac{N}{l+1}\right) \underline{\Theta}_{\frac{N}{l}-1}} d t
$$

for $k \geq 1, \quad l \leq \frac{N}{2}-1$,

$$
\frac{1}{6 \pi} \int_{\frac{N}{l+1}}^{\frac{N}{l}} \frac{\cos \frac{k \pi}{l+1}+1}{\frac{N}{k \pi} \sin \frac{k \pi}{l+1}+t-\frac{N}{l+1}} \cdot \frac{\frac{1}{k} \bar{\Psi}_{k\left(\frac{N}{l+1}-1\right)}^{f\left[k\left(\frac{N}{l+1}+1\right)\right]}}{\frac{1}{k+1} \underline{\Psi}_{0}^{k+1}+\frac{1}{k} \bar{\Psi}_{k}^{k\left(\frac{N}{l+1}-1\right)}+\left(t-\frac{N}{l+1}\right) \bar{\Theta}_{k\left(\frac{N}{l}-1\right)}} d t ;
$$


and for $k \geq 1, \quad \frac{N}{2} \leq l \leq N-2$,

$$
\frac{1}{6 \pi} \int_{\frac{N}{l+1}}^{\frac{N}{l}} \frac{\cos \frac{k \pi}{l+1}+1}{\frac{N}{k \pi} \sin \frac{k \pi}{l+1}+t-\frac{N}{l+1}} \cdot \frac{\frac{1}{k} \bar{\Psi}_{k\left(\frac{N}{l+1}-1\right)}^{f\left[k\left(\frac{N}{l+1}+1\right)\right]}}{\frac{1}{k+1} \underline{\Psi}_{0}^{(k+1)\left(\frac{N}{l+1}-1\right)}+\left(t-\frac{N}{l+1}\right) \underline{\Theta}_{(k+1)\left(\frac{N}{l}-1\right)}} d t .
$$

We have thus defined $\bar{J}_{k}(\bar{\theta}, \underline{\theta})$.

Similarly we have

$$
\begin{aligned}
\theta(s) & \geq \frac{1}{6 \pi} \int_{0}^{\pi} \cot \frac{t}{2} \log \left(1+\frac{\int_{|s-t|}^{\pi-|s+t-\pi|} \underline{\Theta}\left(\frac{(k+1) w}{s}\right) d w}{\int_{0}^{\min (s,|s-t|)} \bar{\Theta}\left(\frac{k w}{s}\right) d w+\int_{\min (s,|s-t|)}^{|s-t|}\left(\frac{(k+1) w}{s}\right) d w}\right) d t \\
& =\frac{1}{6 \pi} \int_{0}^{\frac{\pi}{s}} \frac{s}{\tan \frac{s t}{2}} \log \left(1+\frac{\int_{|t-1|}^{\frac{\pi}{s}-\left|1+t-\frac{\pi}{s}\right|} \underline{\Theta}((k+1) w) d w}{\int_{0}^{\min (1,|t-1|)} \bar{\Theta}(k w) d w+\int_{\min (1,|t-1|)}^{|t-1|}((k+1) w) d w}\right) d t \\
& \geq \frac{1}{6 \pi} \int_{0}^{\frac{N}{k+1}} \frac{\frac{(k+1) \pi}{N}}{\tan \frac{(k+1) t \pi}{2 N}} \log \left(1+\frac{\int_{|t-1|}^{\frac{N}{k+1}-\left|1+t-\frac{N}{k+1}\right|} \underline{\Theta}((k+1) w) d w}{\int_{0}^{\min (1,|t-1|)} \bar{\Theta}(k w) d w+\int_{\min (1,|t-1|)}^{|t-1|}((k+1) w) d w}\right) d t \\
& \geq \sum_{l=0}^{N-1} \underline{A}_{k, l}(\underline{\theta}, \bar{\theta})+\sum_{l=k+1}^{N-1} \max \left(\underline{B}_{k, l}(\underline{\theta}, \bar{\theta}), \underline{C}_{k, l}(\underline{\theta}, \bar{\theta})\right) \equiv \underline{J}_{k}(\underline{\theta}, \bar{\theta}),
\end{aligned}
$$

where $\underline{A}_{k, l}, \underline{B}_{k, l}$ and $\underline{C}_{k, l}$ are defined as follows:

for $k=0, \underline{A}_{k, l}(\underline{\theta}, \bar{\theta})$ is defined as

$\frac{1}{6 \pi} \int_{\frac{l}{N}}^{\frac{l+1}{N}} \frac{\frac{\pi}{N}}{\tan \frac{(l+1) \pi}{2 N^{2}}} \log \left(1+\frac{\max \left(\underline{\Psi}_{1-\frac{l}{N}}^{1+\frac{l}{N}}, \frac{2(l+1)}{N} \underline{\Theta}_{1+\frac{l+1}{N}}\right)}{(1-t) \bar{\Theta}_{0}}\right) d t$

and for $k \geq 1$,

$\frac{1}{6 \pi} \int_{\frac{l}{N}}^{\frac{l+1}{N}} \frac{\frac{(k+1) \pi}{N}}{\tan \frac{(k+1)(l+1) \pi}{2 N^{2}}} \log \left(1+\frac{\max \left(\frac{1}{k+1} \underline{\Psi}_{(k+1)\left(1-\frac{l}{N}\right)}^{f\left[(k+1)\left(1+\frac{l}{N}\right)\right],} A\right)}{\frac{1}{k} \bar{\Psi}_{0}^{k\left(1-\frac{l+1}{N}\right)}+\left(\frac{l+1}{N}-t\right) \bar{\Theta}_{k\left(1-\frac{l+1}{N}\right)}}\right) d t$

where we have put $A=2 \min \left(\frac{l+1}{N}, \frac{N}{k+1}-1\right) \cdot \underline{\Theta}_{\min }\left((k+1)\left(1+\frac{l+1}{N}\right), N, 2 N-(k+1)\left(1+\frac{l}{N}\right)\right)$. 
$\underline{B}_{k, l}(\underline{\theta}, \bar{\theta}) \quad$ is defined as $\quad 0 \quad$ for $\quad l=k+1$;

for $k=0, \quad k+2 \leq l \leq \frac{N}{2}-1$,

$\frac{1}{6 \pi} \int_{\frac{N}{l+1}}^{\frac{N}{l}} \frac{\frac{\pi}{N}}{\tan \frac{\pi}{2 l}} \log \left(1+\frac{\underline{\Psi}_{\frac{N}{l}-1}^{\frac{N}{l}+1}}{\bar{\Theta}_{0}+\underline{\Psi}_{1}^{\frac{N}{l+1}-1}+\left(t-\frac{N}{l+1}\right) \underline{\Theta}_{\frac{N}{l+1}}-1}\right) d t$

for $k=0, \quad \frac{N}{2} \leq l$,

$\frac{1}{6 \pi} \int_{\frac{N}{l+1}}^{\frac{N}{l}} \frac{\frac{\pi}{N}}{\tan \frac{\pi}{2 l}} \log \left(1+\frac{\underline{\Psi}_{\frac{N}{l}-1}^{\frac{N}{l}+1}}{(t-1) \bar{\Theta}_{0}}\right) d t$

for $k \geq 1, \quad k+2 \leq l \leq \frac{N}{2}-1$,

$\frac{1}{6 \pi} \int_{\frac{N}{l+1}}^{\frac{N}{l}} \frac{\frac{(k+1) \pi}{N}}{\tan \frac{(k+1) \pi}{2 l}} \log \left(1+\frac{\frac{1}{k+1} \underline{\Psi}_{(k+1)\left(\frac{N}{l}-1\right)}^{f\left[(k+1)\left(\frac{N}{l}+1\right)\right]}}{\frac{1}{k} \bar{\Psi}_{0}^{k}+\frac{1}{k+1} \underline{\Psi}_{k+1}^{(k+1)\left(\frac{N}{l+1}-1\right)}+\left(t-\frac{N}{l+1}\right) \underline{\Theta}_{(k+1)\left(\frac{N}{l+1}-1\right)}}\right) d t$

and for $k \geq 1, \quad \frac{N}{2} \leq l$,

$\frac{1}{6 \pi} \int_{\frac{N}{l+1}}^{\frac{N}{l}} \frac{\frac{(k+1) \pi}{N}}{\tan \frac{(k+1) \pi}{2 l}} \log \left(1+\frac{\frac{1}{k+1} \underline{\Psi}_{(k+1)\left(\frac{N}{l}-1\right)}^{f\left[(k+1)\left(\frac{N}{l}+1\right)\right]}}{\frac{1}{k} \bar{\Psi}_{0}^{k\left(\frac{N}{l+1}-1\right)}+\left(t-\frac{N}{l+1}\right) \bar{\Theta}_{k\left(\frac{N}{l+1}-1\right)}}\right) d t$.

$\underline{C}_{k, l}(\underline{\theta}, \bar{\theta})$ is defined as 0 for $l=k+1$;

for $k=0, k+2 \leq l \leq \frac{N}{2}-1$,

$\frac{1}{6 \pi} \int_{\frac{N}{l+1}}^{\frac{N}{l}} \frac{\frac{\pi}{l(l+1)}}{\left(t-\frac{N}{l+1}\right) \tan \frac{\pi}{2 l}+\left(\frac{N}{l}-t\right) \tan \frac{\pi}{2(l+1)}}$

$\times \frac{\bar{\Theta}_{0}+\underline{\Psi}_{1}^{\frac{N}{l+1}-1}}{\bar{\Theta}_{0}+\underline{\Psi}_{1}^{\frac{N}{l+1}-1}+\left(t-\frac{N}{l+1}\right) \underline{\Theta}_{\frac{N}{l+1}-1}} \log \left(1+\frac{\underline{\Psi}_{\frac{N}{l}-1}^{\frac{N}{l}+1}}{\bar{\Theta}_{0}+\underline{\Psi}_{1}^{\frac{N}{l+1}-1}}\right) d t$

for $k=0, \quad \frac{N}{2} \leq l$,

$\frac{1}{6 \pi} \int_{\frac{N}{l+1}}^{\frac{N}{l}} \frac{\frac{\pi}{l(l+1)}}{\left(t-\frac{N}{l+1}\right) \tan \frac{\pi}{2 l}+\left(\frac{N}{l}-t\right) \tan \frac{\pi}{2(l+1)}} \cdot \frac{\left(\frac{N}{l+1}-1\right) \bar{\Theta}_{0}}{(t-1) \bar{\Theta}_{0}} \log \left(1+\frac{\underline{\Psi}_{\frac{N}{l}-1}^{\frac{N}{l}+1}}{\left(\frac{N}{l+1}-1\right) \bar{\Theta}_{0}}\right) d t$ 
for $k \geq 1, \quad k+2 \leq l \leq \frac{N}{2}-1$,

$$
\begin{aligned}
& \frac{1}{6 \pi} \int_{\frac{N}{l+1}}^{\frac{N}{l}} \frac{\frac{(k+1) \pi}{l(l+1)}}{\left(t-\frac{N}{l+1}\right) \tan \frac{(k+1) \pi}{2 l}+\left(\frac{N}{l}-t\right) \tan \frac{(k+1) \pi}{2(l+1)}} \\
& \times \frac{\frac{1}{k} \bar{\Psi}_{0}^{k}+\frac{1}{k+1} \underline{\Psi}_{k+1}^{(k+1)\left(\frac{N}{l+1}-1\right)}}{\frac{1}{k} \bar{\Psi}_{0}^{k}+\frac{1}{k+1} \underline{\Psi}_{k+1}^{(k+1)\left(\frac{N}{l+1}-1\right)}+\left(t-\frac{N}{l+1}\right) \underline{\Theta}_{(k+1)\left(\frac{N}{l+1}-1\right)}} \\
& \times \log \left(1+\frac{\frac{1}{k+1} \underline{\Psi}_{(k+1)\left(\frac{N}{l}-1\right)}^{f\left[(k+1)\left(\frac{N}{l}+1\right)\right]}}{\frac{1}{k} \bar{\Psi}_{0}^{k}+\frac{1}{k+1} \underline{\Psi}_{k+1}^{(k+1)\left(\frac{N}{l+1}-1\right)}}\right) d t
\end{aligned}
$$

and for $k \geq 1, \quad \frac{N}{2} \leq l$,

$$
\begin{aligned}
\frac{1}{6 \pi} \int_{\frac{N}{l+1}}^{\frac{N}{l}} \frac{\frac{(k+1) \pi}{l(l+1)}}{\left(t-\frac{N}{l+1}\right) \tan \frac{(k+1) \pi}{2 l}+\left(\frac{N}{l}-t\right) \tan \frac{(k+1) \pi}{2(l+1)}} & \\
& \quad \times \frac{\frac{1}{k} \bar{\Psi}_{0}^{k\left(\frac{N}{l+1}-1\right)}}{\frac{1}{k} \bar{\Psi}_{0}^{k\left(\frac{N}{l+1}-1\right)}+\left(t-\frac{N}{l+1}\right) \bar{\Theta}_{k\left(\frac{N}{l+1}-1\right)}} \log \left(1+\frac{\frac{1}{k+1} \underline{\Psi}_{(k+1)\left(\frac{N}{l}-1\right)}^{f\left[(k+1)\left(\frac{N}{l}+1\right)\right]}}{\frac{1}{k} \bar{\Psi}_{0}^{k\left(\frac{N}{l+1}-1\right)}}\right) d t .
\end{aligned}
$$

Next, note that

$$
\begin{array}{r}
G(\bar{\theta}, \underline{\theta}, g)(s)=\frac{1}{6 \pi} \int_{0}^{\pi} \cot \frac{t}{2} \times\left(\frac{\int_{|s-t|}^{\pi-|s+t-\pi|} \sin \bar{\theta}(w) d w \cdot \int_{0}^{\min (s,|s-t|)} \cos \underline{\theta}(w) \cdot g(w) d w}{\int_{0}^{|s-t|} \sin \underline{\theta}(w) d w}\right. \\
\left.\quad+\int_{|s-t|}^{\pi-|s+t-\pi|} \cos \underline{\theta}(w) \cdot g(w) d w\right) \times \frac{d t}{\int_{0}^{\pi-|s+t-\pi|} \sin \underline{\theta}(w) d w} \\
=\frac{1}{6 \pi} \int_{0}^{\frac{\pi}{s}} \frac{s}{\tan \frac{s t}{2}} \times\left(\frac{\int_{|1-t|}^{\frac{\pi}{s}-\left|1+t-\frac{\pi}{s}\right|} \sin \bar{\theta}(s w) d w \cdot \int_{0}^{\min (1,|1-t|)} \cos \underline{\theta}(s w) \cdot g(s w) d w}{\int_{0}^{|1-t|} \sin \underline{\theta}(s w) d w}\right. \\
\left.\quad+\int_{|1-t|}^{\frac{\pi}{s}-\left|1+t-\frac{\pi}{s}\right|} \cos \underline{\theta}(s w) \cdot g(s w) d w\right) \times \frac{d t}{\int_{0}^{\frac{\pi}{s}-\left|1+t-\frac{\pi}{s}\right|} \sin \underline{\theta}(s w) d w}
\end{array}
$$




$$
\begin{aligned}
& \leq \frac{1}{6 \pi} \int_{0}^{1} \lim _{\sigma \downarrow \frac{k \pi}{N}} \frac{\sigma}{\tan \frac{\sigma t}{2}} \times\left\{\min \left(\frac{\int_{1-t}^{\frac{\pi}{\sigma}-\left|1+t-\frac{\pi}{\sigma}\right|} \bar{\Theta}(k w) d w}{\int_{0}^{\frac{N}{k+1}-\left|1+t-\frac{N}{k+1}\right|} \underline{\Theta}((k+1) w) d w}, c_{1}\right)\right. \\
& \left.\times \min \left(\frac{\int_{0}^{1-t} \gamma(k w) d w}{\left.\int_{0}^{1-t} \underline{\Theta}(k+1) w\right) d w}, c_{2}\right)+\min \left(\frac{\int_{1-t}^{\frac{\pi}{\sigma}-\left|1+t-\frac{\pi}{\sigma}\right|} \gamma(k w) d w}{\left.\int_{0}^{\frac{N}{k+1}-\left|1+t-\frac{N}{k+1}\right|} \underline{\Theta}(k+1) w\right) d w}, c_{2}\right)\right\} d t \\
& +\frac{1}{6 \pi} \int_{1}^{\frac{N}{k}} \lim _{\sigma \downarrow \frac{k \pi}{N}} \frac{\sigma}{\tan \frac{\sigma t}{2}} \times\left\{\min \left(\frac{\int_{t-1}^{\frac{\pi}{\sigma}-\left|1+t-\frac{\pi}{\sigma}\right|} \bar{\Theta}(k w) d w}{\int_{0}^{\max \left(\frac{N}{k+1}-\left|1+t-\frac{N}{k+1}\right|, 0\right)} \underline{\Theta}((k+1) w) d w}, c_{1}, \frac{2 \bar{\Theta}(0)}{1+t} c_{3}\right)\right. \\
& \times \min \left(\frac{\int_{0}^{\min (1, t-1)} \gamma(k w) d w}{\int_{0}^{t-1} \underline{\Theta}((k+1) w) d w}, c_{2}\right) \\
& \left.+\min \left(\frac{\int_{t-1}^{\frac{\pi}{\sigma}-\left|1+t-\frac{\pi}{\sigma}\right|} \gamma(k w) d w}{\int_{0}^{\max \left(\frac{N}{k+1}-\left|1+t-\frac{N}{k+1}\right|, 0\right)} \underline{\Theta}((k+1) w) d w}, c_{2}, \frac{2 \gamma(0)}{1+t} c_{3}\right)\right\} d t \\
& \leq \sum_{l=0}^{N-1} D_{k, l}(\bar{\theta}, \underline{\theta}, g)+\sum_{l=k}^{N-1} \min \left(E_{k, l}(\bar{\theta}, \underline{\theta}), F_{k, l}(\bar{\theta}, \underline{\theta})\right) \equiv G_{k}(\bar{\theta}, \underline{\theta}, g),
\end{aligned}
$$

where $D_{k, l}, E_{k, l}$ and $F_{k, l}$ are defined as follows:

for $\quad k=l=0, D_{k, l}(\bar{\theta}, \underline{\theta}, g)$ is defined as

$\frac{1}{6 \pi} \int_{0}^{\frac{1}{N}} \frac{4}{\underline{\Psi}_{0}^{1}}\left\{\bar{\Theta}_{0} \cdot \min \left(\frac{\gamma_{0}}{\underline{\Psi}_{0}^{1-\frac{1}{N}}}, c_{2}\right)+\gamma_{0}\right\} d t$

for $k=0, \quad l \geq 1$,

$\frac{1}{6 \pi} \int_{\frac{l}{N}}^{\frac{l+1}{N}} \frac{2 N}{l}\left\{\min \left(\frac{\frac{2 l}{N} \bar{\Theta}_{0}}{\underline{\Psi}_{0}^{1+\frac{l}{N}}}, c_{1}\right) \cdot \min \left(\frac{\left(1-\frac{l}{N}\right) \gamma_{0}}{\underline{\Psi}_{0}^{1-\frac{l+1}{N}}}, c_{2}\right)+\min \left(\frac{\frac{2 l}{N} \gamma_{0}}{\underline{\Psi}_{0}^{1+\frac{l}{N}}}, c_{2}\right)\right\} d t ;$

for $k \geq 1, \quad l=0$

$$
\begin{aligned}
\frac{1}{6 \pi} \int_{0}^{\frac{1}{N}} \frac{4}{\frac{1}{k+1} \underline{\Psi}_{0}^{\min \left(k+1,2 N-(k+1)\left(1+\frac{1}{N}\right)\right)}} \\
\quad \times\left\{\overline { \Theta } _ { k ( 1 - \frac { 1 } { N } ) } \cdot \operatorname { m i n } \left(\frac{\frac{1}{k} \Gamma_{0}^{k}}{\left.\left.\frac{1}{k+1} \underline{\Psi}_{0}^{(k+1)\left(1-\frac{1}{N}\right)}, c_{2}\right)+\gamma_{k\left(1-\frac{1}{N}\right)}\right\} d t ;}\right.\right.
\end{aligned}
$$


and for $k \geq 1, \quad l \geq 1$,

$$
\begin{aligned}
\frac{1}{6 \pi} \int_{\frac{l}{N}}^{\frac{l+1}{N}} \frac{\frac{k \pi}{N}}{\tan \frac{k l \pi}{2 N^{2}}}\left\{\operatorname { m i n } \left(\frac{\min \left(\frac{2 l}{N} \bar{\Theta}_{k\left(1-\frac{l+1}{N}\right)}, \frac{1}{k} \bar{\Psi}_{k\left(1-\frac{l+1}{N}\right)}^{f\left[k\left(1+\frac{l+1}{N}\right)\right]}\right)}{\left.\frac{1}{k+1} \underline{\Psi}_{0}^{\min \left((k+1)\left(1+\frac{l}{N}\right), 2 N-(k+1)\left(1+\frac{l+1}{N}\right)\right)}, c_{1}\right)}\right.\right. \\
\times \min \left(\frac{\frac{1}{k} \Gamma_{0}^{k\left(1-\frac{l}{N}\right)}}{\left.\frac{1}{k+1} \underline{\Psi}_{0}^{(k+1)\left(1-\frac{l+1}{N}\right)}, c_{2}\right)}\right. \\
\left.+\min \left(\frac{\min \left(\frac{2 l}{N} \gamma_{k\left(1-\frac{l+1}{N}\right)}, \frac{1}{k} \Gamma_{k\left(1-\frac{l+1}{N}\right)}^{f\left[k\left(1+\frac{l+1}{N}\right)\right]}\right)}{\frac{1}{k+1} \underline{\Psi}_{0}^{\min \left((k+1)\left(1+\frac{l}{N}\right), 2 N-(k+1)\left(1+\frac{l+1}{N}\right)\right)}, c_{2}}\right)\right\} d t .
\end{aligned}
$$

$E_{k, l}(\bar{\theta}, \underline{\theta}, g)$ is defined as $\infty$ for $l=0$;

for $k=0, \quad l \geq 1$,

$$
\begin{array}{r}
\frac{1}{6 \pi} \int_{\frac{N}{l+1}}^{\frac{N}{l}} \frac{2(l+1)}{N}\left\{\operatorname { m i n } \left(\frac{2 \bar{\Theta}_{0}}{\left.\underline{\Psi}_{0}^{1+\frac{N}{l+1}}, c_{1}\right) \cdot \min \left(\frac{\min \left(1, \frac{N}{l}-1\right) \gamma_{0}}{\underline{\Psi}_{0}^{\frac{N}{l+1}-1}}, c_{2}\right)}\right.\right. \\
\left.+\min \left(\frac{2 \gamma_{0}}{\underline{\Psi}_{0}^{1+\frac{N}{l+1}}}, c_{2}\right)\right\} d t ;
\end{array}
$$

and for $k \geq 1, \quad l \geq 1$,

$$
\begin{aligned}
\frac{1}{6 \pi} \int_{\frac{N}{l+1}}^{\frac{N}{l}} \frac{\frac{k \pi}{N}}{\tan \frac{k \pi}{2(l+1)}} & \left\{\operatorname { m i n } \left(\frac{\frac{1}{k} \bar{\Psi}_{k\left(\frac{N}{l+1}-1\right)}^{f\left[k\left(1+\frac{N}{l+1}\right)\right]}}{\left.\frac{1}{k+1} \underline{\Psi}_{0}^{\min \left((k+1)\left(1+\frac{N}{l+1}\right), \max \left(2 N-(k+1)\left(1+\frac{N}{l}\right), 0\right)\right)}, c_{1}\right)}\right.\right. \\
& \times \min \left(\frac{\frac{1}{k} \Gamma_{0}^{k \min \left(1, \frac{N}{l}-1\right)}}{\left.\frac{1}{k+1} \underline{\Psi}_{0}^{(k+1)\left(\frac{N}{l+1}-1\right)}, c_{2}\right)}\right. \\
& \left.+\min \left(\frac{\frac{1}{k} \Gamma_{k\left(\frac{N}{l+1}-1\right)}^{f\left[k\left(1+\frac{N}{l+1}\right)\right]}}{\frac{1}{k+1} \underline{\Psi}_{0}^{\min \left((k+1)\left(1+\frac{N}{l+1}\right), \max \left(2 N-(k+1)\left(1+\frac{N}{l}\right), 0\right)\right)}, c_{2}}\right)\right\} d t .
\end{aligned}
$$

$F_{k, l}(\bar{\theta}, \underline{\theta}, g)$ is defined for $k=0$ as,

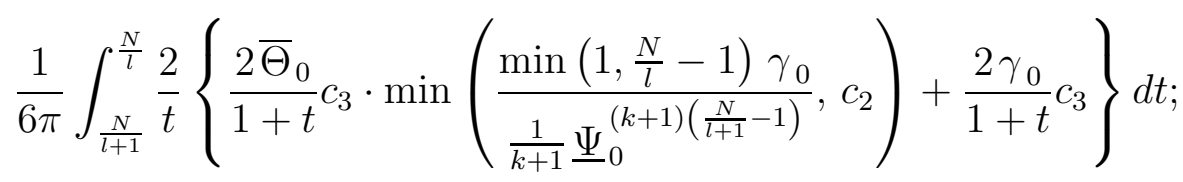


and for $k>0$,

$$
\frac{1}{6 \pi} \int_{\frac{N}{l+1}}^{\frac{N}{l}} \frac{\cos \frac{k \pi}{l+1}+1}{\frac{N}{k \pi} \sin \frac{k \pi}{l+1}+t-\frac{N}{l+1}}\left\{\frac{2 \bar{\Theta}_{0}}{1+t} c_{3} \cdot \min \left(\frac{\frac{1}{k} \Gamma_{0}^{k \min \left(1, \frac{N}{l}-1\right)}}{\frac{1}{k+1} \underline{\Psi}_{0}^{(k+1)\left(\frac{N}{l+1}-1\right)}}, c_{2}\right)+\frac{2 \gamma_{0}}{1+t} c_{3}\right\} d t
$$

The inequalities (3.2)-(3.4) are verified elementarily, though the proof is lengthy. In the proof, we use the following facts.

1. $\frac{x}{\tan x y} \log (1+c y), \quad(x>0, y>0, x y<\pi / 2, c:$ a positive constant $)$

is monotone decreasing in $x$ and $y$.

2. $x-|c-x|$ is a non-decreasing function of $x$.

3. For an arbitrary non-increasing and non-negative function $P(w)$ and a positive constant $k \leq n$,

$\int_{k(1-x)}^{f[k(1+x)]} P(w) d w$ is non-decreasing in $0 \leq x \leq 1$.

4. For an arbitrary non-increasing and non-negative function $P(w)$ and a positive constant $k \leq n$,

$\int_{k(x-1)}^{f[k(1+x)]} P(w) d w$ is non-increasing in $1 \leq x$.

5. $\frac{\log (1+\bar{x})}{\bar{x}} \cdot x \leq \log (1+x) \leq x$, for $0<x \leq \bar{x}$.

6. For an arbitrary non-increasing and non-negative function $P(w)$, a positive constant $c \geq 1$ and $x \in[1, c]$,

$$
\begin{aligned}
& \frac{\int_{x-1}^{c-|1+x-c|} P(w) d w}{c-|1+x-c|} \leq \frac{c-|1+x-c|-(x-1)}{c-|1+x-c|} \cdot P(0) \leq \frac{(1+x)-(x-1)}{1+x} \cdot P(0)= \\
& \frac{2 P(0)}{1+x} \text {. }
\end{aligned}
$$

\section{Numerical result}

The IEEE754 standard defines several modes for controlling the behavior of floating point operations. We can choose a rounding mode: round-up, round-down, or roundto-nearest (see [6], [9]). In our computation, we use both round-up and round-down appropriately to obtain rigorous mathematical results. We used a Pentium4 PC with Red Hat Linux 8.0 and gcc.

We control rounding mode to make $\bar{J}_{*}$ and $G_{*}$ as large as possible, and $\underline{J}_{*}$ as small as possible. 
Set $N=3000$, and we calculate following sequence of functions,

$$
\left\{\begin{aligned}
\bar{\theta}_{0}(s) & =\frac{\pi}{2}, \\
\underline{\theta}_{0}(s) & =0.00005 \cdot 1_{0<s \leq \pi / 2}, \\
\bar{\theta}_{n+1}(s) & =\min \left(\bar{\theta}_{n}(s), \bar{J}_{*}\left(\bar{\theta}_{n}, \underline{\theta}_{n}\right)(s)\right), \\
\underline{\theta}_{n+1}(s) & =\max \left(\underline{\theta}_{n}(s), \underline{J}_{*}\left(\underline{\theta}_{n}, \bar{\theta}_{n}\right)(s)\right) .
\end{aligned}\right.
$$

From (3.2),(3.3) and Theorem 2.2, any solution of (1.1) must lie between $\bar{\theta}_{n}(s)$ and $\underline{\underline{\theta}} n$.

$\overline{\bar{\theta}}_{n}^{n}(s)-\underline{\theta}_{n}(s)$ decreases as $n$ grows (Fig.1). The shape of $\bar{\theta}_{0}(s), \bar{\theta}_{5}(s), \bar{\theta}_{10}(s), \cdots, \bar{\theta}_{45}(s)$ and

$\underline{\theta}_{0}(s), \underline{\theta}_{5}(s), \underline{\theta}_{10}(s), \cdots, \underline{\theta}_{45}(s)$ are shown in Fig.2, Fig.3 and Fig.4.

To prove uniqueness, we calculate the following sequence of functions,

$$
\left\{\begin{aligned}
g_{0}(s) & =1, \\
g_{n+1}(s) & =\frac{\bar{G}\left(\bar{\theta}_{45}, \underline{\theta}_{45}, g_{n}\right)(s)}{\sup _{0<s \leq \pi} \bar{G}\left(\bar{\theta}_{45}, \underline{\theta}_{45}, g_{n}\right)(s)},
\end{aligned}\right.
$$

and the following inequality was verified:

$$
\sup _{0<s \leq \pi} \frac{\bar{G}\left(\bar{\theta}_{45}, \underline{\theta}_{45}, g_{4}\right)(s)}{g_{4}(s)}=0.99865799 \cdots<1 .
$$

Then, Theorem 2.3 and the results of previous section implies that the solution of (1.1), namely Stokes' wave of extreme form, is globally unique.

The shape of $g_{4}(s)$ is shown in Fig.5. The whole calculation took approximately 815 minutes.

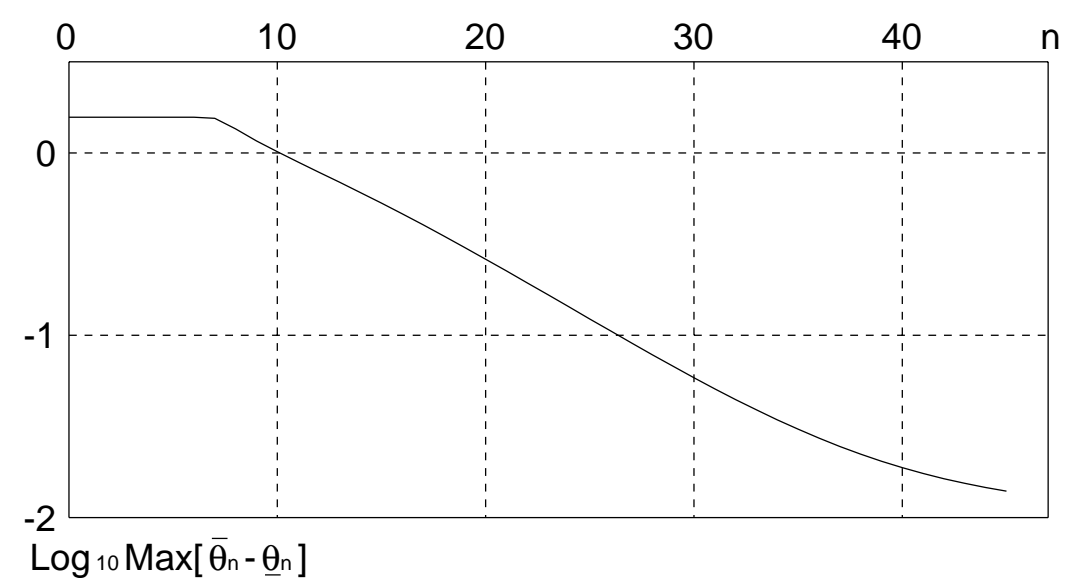

Figure 1: $n$ vs. $\log _{10} \max _{s}\left(\bar{\theta}_{n}(s)-\underline{\theta}_{n}(s)\right)$. 


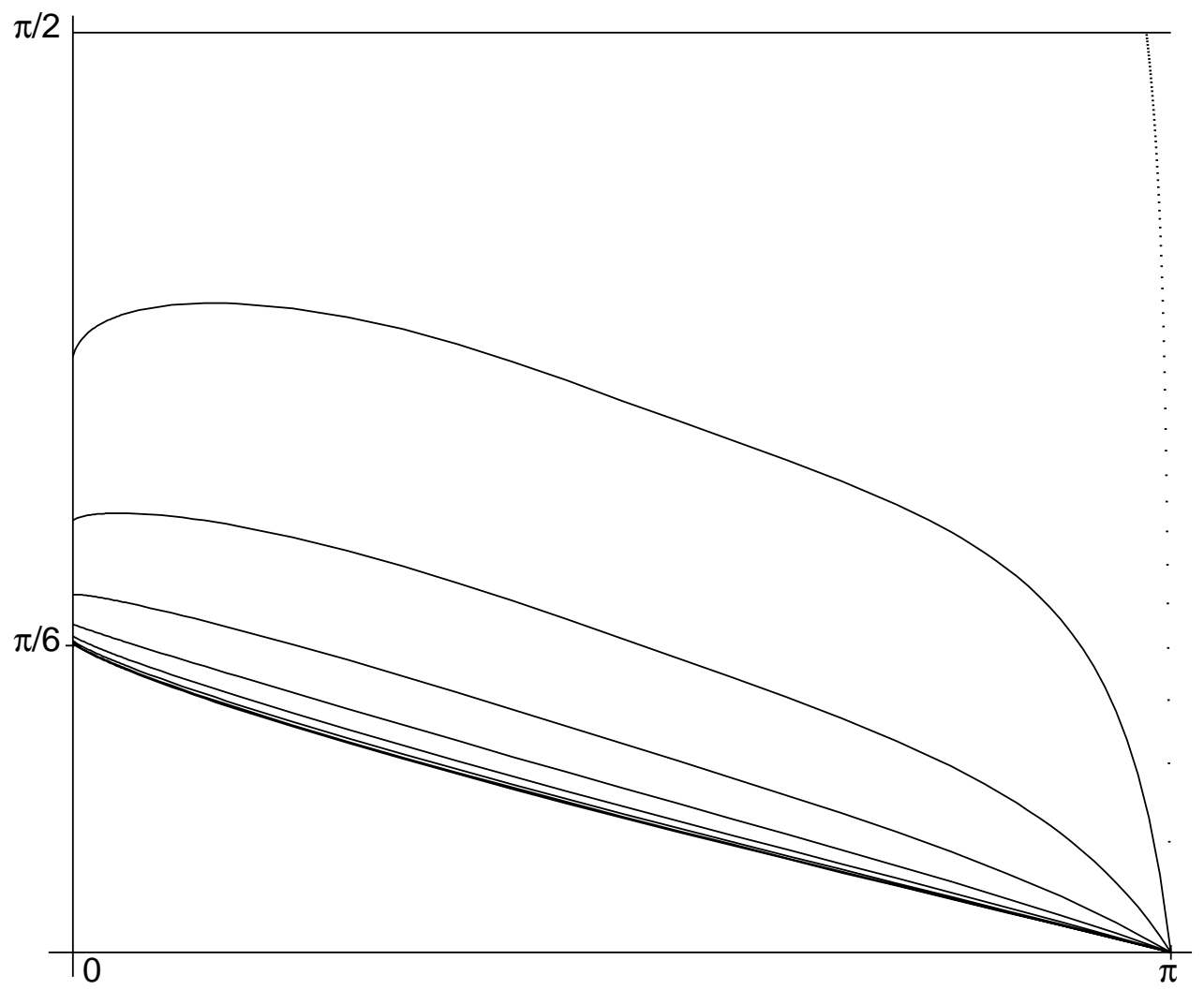

Figure 2: The shape of $\bar{\theta}_{0}(s), \bar{\theta}_{5}(s), \cdots, \bar{\theta}_{40}(s)$ and $\bar{\theta}_{45}(s)$.

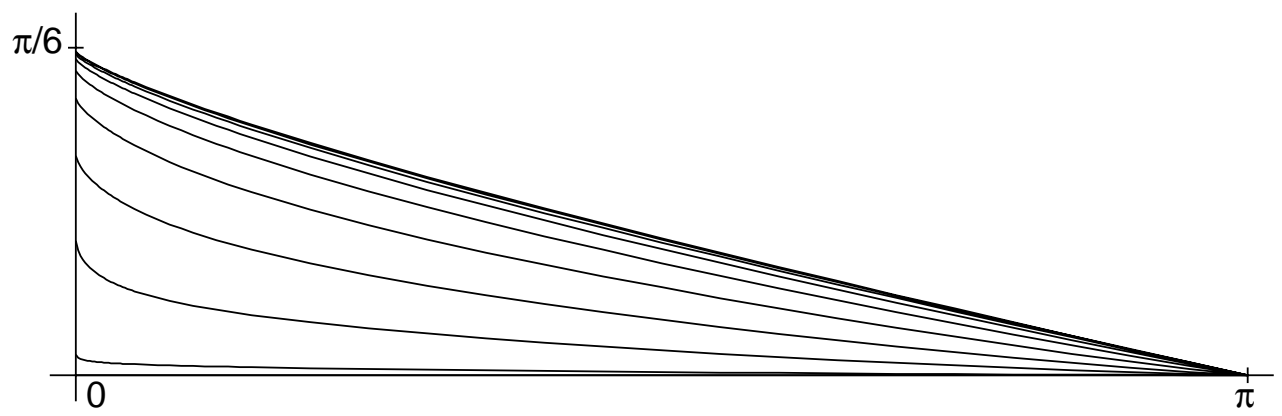

Figure 3: The shape of $\underline{\theta}_{0}(s), \underline{\theta}_{5}(s), \cdots, \underline{\theta}_{40}(s)$ and $\underline{\theta}_{45}(s)$. 


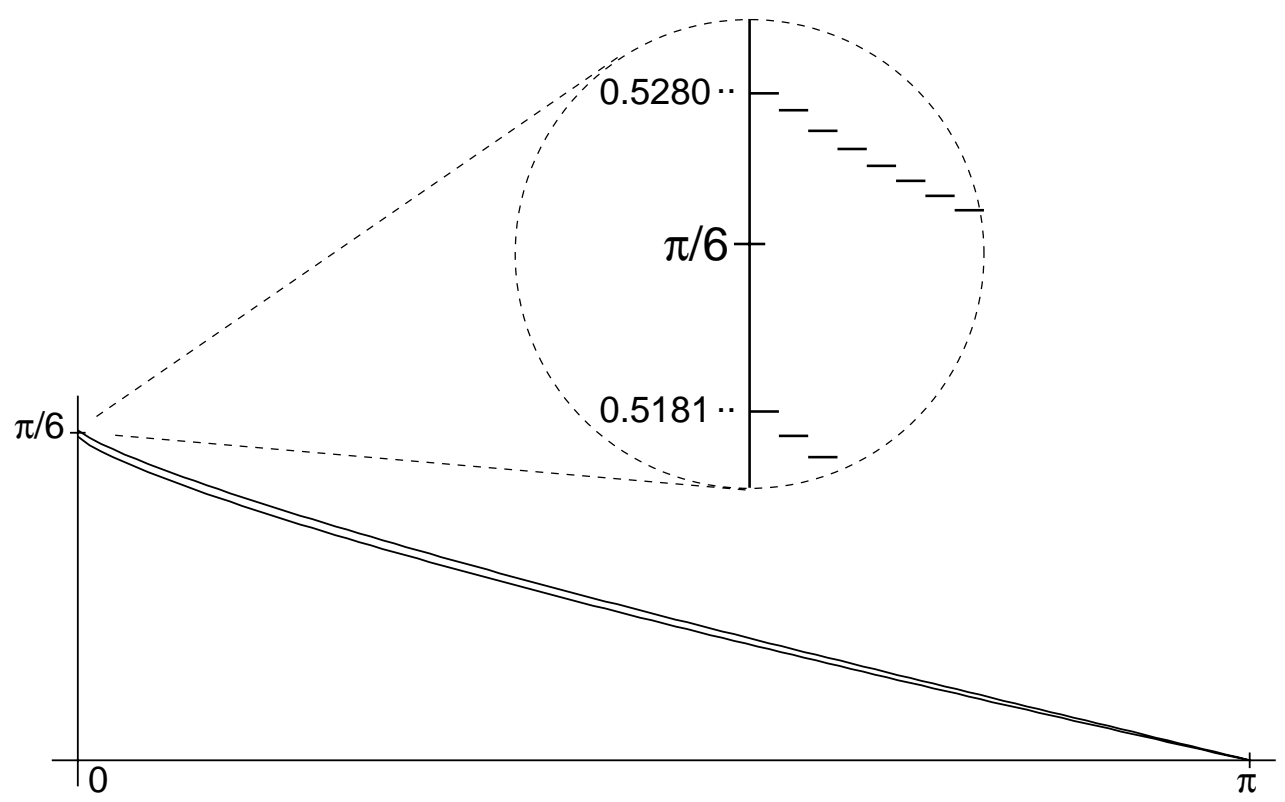

Figure 4: The shape of $\bar{\theta}_{45}(s)$ and $\underline{\theta}_{45}(s)$.

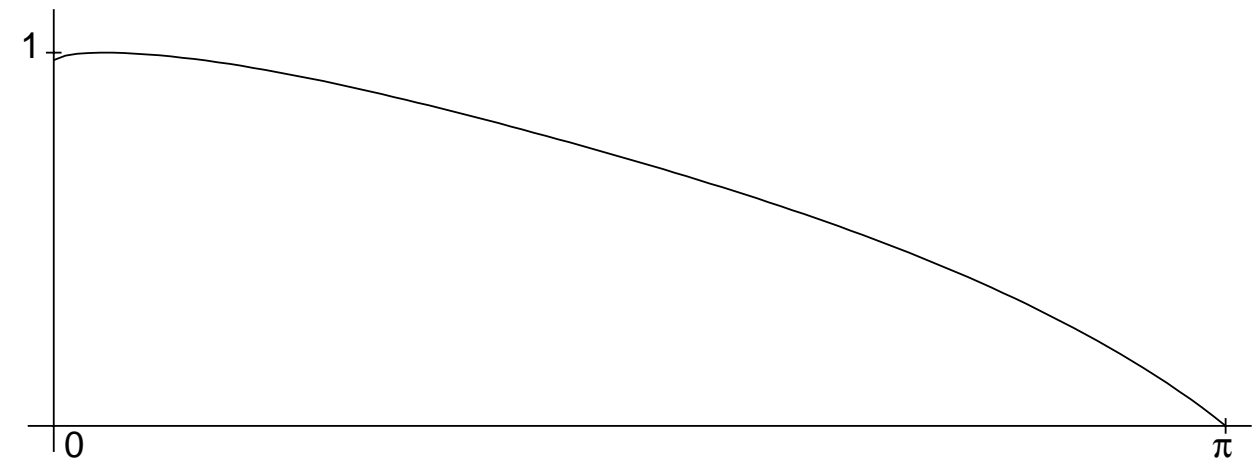

Figure 5: The shape of $g_{4}(s)$.

In order for our computer program to be openly tested, the author is ready to send his program on request. Also, it is posted in his internet homepage.

\section{Conclusion}

We have proved the global uniqueness of Stokes' wave of extreme form by the numerical verification method. Thus, combined with the results of [12] and [13], the second Stokes conjecture (the conjecture (b) of section 1) is solved affirmatively. Besides, our method seems to have applications to other nonlinear problems.

\section{Acknowledgment}

I would like to thank Professor H. Okamoto of Kyoto University for helpful discussion and advice. This paper was completed during the author's stay in Kyushu 


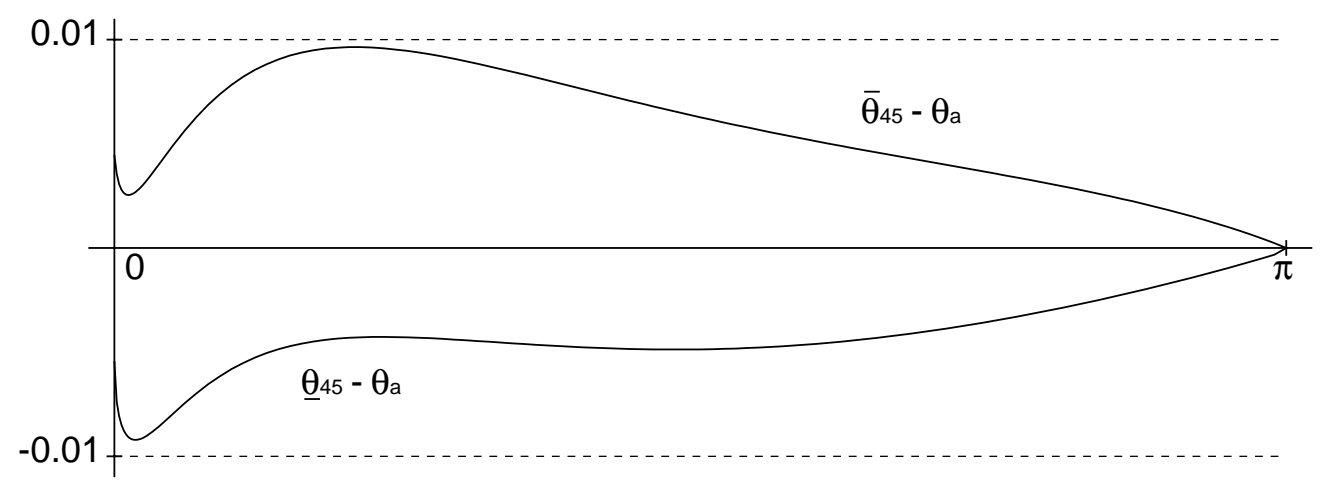

Figure 6: $\bar{\theta}_{45}(s)-\theta_{a}(s)$ and $\underline{\theta}_{45}(s)-\theta_{a}(s)$

University as a JSPS post-doctoral fellow. He is very grateful to Professor M. T. Nakao for his hospitality. This research is partly supported by the Grant-in-Aid for JSPS Fellows, The Ministry of Education, Culture, Sports, Science and Technology, Japan and the 21st Century COE program of Faculty of Mathematics, Kyushu University.

\section{Reference}

[1] Amick, C.J., Fraenkel, L.E. \& Toland, J.F. (1982) On the Stokes' conjecture for the wave of extreme form, Acta Math., 148, 193-214.

[2] Fraenkel, L.E. (to appear) A constructive existence proof for the extreme Stokes wave, Arch. Rational. Mech. Anal., ,--

[3] Kobayashi, K. (2004) Numerical verification of the global uniqueness of a positive solution for Nekrasov's equation, Japan J. Indust. Appl. Math., 21 (2), 181218.

[4] Keady, G. \& Norbury, J. (1978) On the existence theory for irrotational water waves, Math. Proc. Camb. Phil. Soc., 83, 137-157.

[5] Krasovskit, Yu.P. (1961) On the theory of steady-state waves of finite amplitude, U.S.S.R. Comput. Math. and Math. Phys., 1, 996-1018. (translation from Russian)

[6] Nakao, M.T. \& Yamamoto, N. (1998) Seido Hoshoutsuki Suuchikeisan (Numerical Computation with Guaranteed Accuracy). Tokyo: Nippon Hyoron Sha. (in Japanese)

[7] Nekrasov, A.I. (1921) On waves of permanent type I, Izv. Ivanovo-Voznesensk. Polite. Inst., 3, 52-65. (in Russian)

[8] Окамото, H. \& ShōJi, M. (2001) The Mathematical Theory of Permanent Progressive Water-Waves. Singapore: World Scientific Publ. Co. 
[9] Rump, S.M. (2001) Self-validating methods, Linear Algebra and its Applications, 324, 3-13.

[10] Sasaki, K. \& Murakami, T. (1973) Irrotational, progressive surface gravity waves near the limiting height, J. Ocean. Soc. Japan, 29, 94-105.

[11] Stokes, G.G. (1880) On the theory of oscillatory waves. Appendix B : Consideration relative to the greatest height of oscillatory irrotational waves which can be propagated without change of form, Math. Phys. Paper, 1, 225-228.

[12] Toland, J.F. (1973) On the existence of waves of greatest height and Stokes' conjecture, Proc. R. Soc. Lond., A 363, 469-485.

[13] Toland, J.F. \& Plotnikov, P.I. (2004) Convexity of Stokes waves of extreme form, Arch. Rat. Mech. Anal., 171, 349-416. 


\section{List of MHF Preprint Series, Kyushu University 21st Century COE Program \\ Development of Dynamic Mathematics with High Functionality}

\section{MHF2005-1 Hideki KOSAKI}

Matrix trace inequalities related to uncertainty principle

MHF2005-2 Masahisa TABATA

Discrepancy between theory and real computation on the stability of some finite element schemes

MHF2005-3 Yuko ARAKI \& Sadanori KONISHI

Functional regression modeling via regularized basis expansions and model selection

MHF2005-4 Yuko ARAKI \& Sadanori KONISHI

Functional discriminant analysis via regularized basis expansions

MHF2005-5 Kenji KAJIWARA, Tetsu MASUDA, Masatoshi NOUMI, Yasuhiro OHTA \& Yasuhiko YAMADA

Point configurations, Cremona transformations and the elliptic difference Painlevé equations

MHF2005-6 Kenji KAJIWARA, Tetsu MASUDA, Masatoshi NOUMI, Yasuhiro OHTA \& Yasuhiko YAMADA

Construction of hypergeometric solutions to the $q$ - Painlevé equations

MHF2005-7 Hiroki MASUDA

Simple estimators for non-linear Markovian trend from sampled data:

I. ergodic cases

MHF2005-8 Hiroki MASUDA \& Nakahiro YOSHIDA

Edgeworth expansion for a class of Ornstein-Uhlenbeck-based models

MHF2005-9 Masayuki UCHIDA

Approximate martingale estimating functions under small perturbations of dynamical systems

MHF2005-10 Ryo MATSUZAKI \& Masayuki UCHIDA

One-step estimators for diffusion processes with small dispersion parameters from discrete observations

MHF2005-11 Junichi MATSUKUBO, Ryo MATSUZAKI \& Masayuki UCHIDA

Estimation for a discretely observed small diffusion process with a linear drift

MHF2005-12 Masayuki UCHIDA \& Nakahiro YOSHIDA

AIC for ergodic diffusion processes from discrete observations 
MHF2005-13 Hiromichi GOTO \& Kenji KAJIWARA

Generating function related to the Okamoto polynomials for the Painlevé IV equation

MHF2005-14 Masato KIMURA \& Shin-ichi NAGATA

Precise asymptotic behaviour of the first eigenvalue of Sturm-Liouville problems with large drift

MHF2005-15 Daisuke TAGAMI \& Masahisa TABATA

Numerical computations of a melting glass convection in the furnace

MHF2005-16 Raimundas VIDŪNAS

Normalized Leonard pairs and Askey-Wilson relations

MHF2005-17 Raimundas VIDŪNAS

Askey-Wilson relations and Leonard pairs

MHF2005-18 Kenji KAJIWARA \& Atsushi MUKAIHIRA

Soliton solutions for the non-autonomous discrete-time Toda lattice equation

MHF2005-19 Yuu HARIYA

Construction of Gibbs measures for 1-dimensional continuum fields

MHF2005-20 Yuu HARIYA

Integration by parts formulae for the Wiener measure restricted to subsets in $\mathbb{R}^{d}$

MHF2005-21 Yuu HARIYA

A time-change approach to Kotani's extension of Yor's formula

MHF2005-22 Tadahisa FUNAKI, Yuu HARIYA \& Mark YOR

Wiener integrals for centered powers of Bessel processes, I

MHF2005-23 Masahisa TABATA \& Satoshi KAIZU

Finite element schemes for two-fluids flow problems

MHF2005-24 Ken-ichi MARUNO \& Yasuhiro OHTA

Determinant form of dark soliton solutions of the discrete nonlinear Schrödinger equation

MHF2005-25 Alexander V. KITAEV \& Raimundas VIDŪNAS

Quadratic transformations of the sixth Painlevé equation

MHF2005-26 Toru FUJII \& Sadanori KONISHI

Nonlinear regression modeling via regularized wavelets and smoothing parameter selection

MHF2005-27 Shuichi INOKUCHI, Kazumasa HONDA, Hyen Yeal LEE, Tatsuro SATO, Yoshihiro MIZOGUCHI \& Yasuo KAWAHARA

On reversible cellular automata with finite cell array 
MHF2005-28 Toru KOMATSU

Cyclic cubic field with explicit Artin symbols

MHF2005-29 Mitsuhiro T. NAKAO, Kouji HASHIMOTO \& Kaori NAGATOU

A computational approach to constructive a priori and a posteriori error estimates for finite element approximations of bi-harmonic problems

MHF2005-30 Kaori NAGATOU, Kouji HASHIMOTO \& Mitsuhiro T. NAKAO Numerical verification of stationary solutions for Navier-Stokes problems

MHF2005-31 Hidefumi KAWASAKI

A duality theorem for a three-phase partition problem

MHF2005-32 Hidefumi KAWASAKI

A duality theorem based on triangles separating three convex sets

MHF2005-33 Takeaki FUCHIKAMI \& Hidefumi KAWASAKI

An explicit formula of the Shapley value for a cooperative game induced from the conjugate point

MHF2005-34 Hideki MURAKAWA

A regularization of a reaction-diffusion system approximation to the two-phase Stefan problem

MHF2006-1 Masahisa TABATA

Numerical simulation of Rayleigh-Taylor problems by an energy-stable finite element scheme

MHF2006-2 Ken-ichi MARUNO \& G R W QUISPEL

Construction of integrals of higher-order mappings

MHF2006-3 Setsuo TANIGUCHI

On the Jacobi field approach to stochastic oscillatory integrals with quadratic phase function

MHF2006-4 Kouji HASHIMOTO, Kaori NAGATOU \& Mitsuhiro T. NAKAO

A computational approach to constructive a priori error estimate for finite element approximations of bi-harmonic problems in nonconvex polygonal domains

MHF2006-5 Hidefumi KAWASAKI

A duality theory based on triangular cylinders separating three convex sets in $R^{n}$

MHF2006-6 Raimundas VIDŪNAS

Uniform convergence of hypergeometric series

MHF2006-7 Yuji KODAMA \& Ken-ichi MARUNO

N-Soliton solutions to the DKP equation and Weyl group actions 
MHF2006-8 Toru KOMATSU

Potentially generic polynomial

MHF2006-9 Toru KOMATSU

Generic sextic polynomial related to the subfield problem of a cubic polynomial

MHF2006-10 Shu TEZUKA \& Anargyros PAPAGEORGIOU

Exact cubature for a class of functions of maximum effective dimension

MHF2006-11 Shu TEZUKA

On high-discrepancy sequences

MHF2006-12 Raimundas VIDŪNAS

Detecting persistent regimes in the North Atlantic Oscillation time series

MHF2006-13 Toru KOMATSU

Tamely Eisenstein field with prime power discriminant

MHF2006-14 Nalini JOSHI, Kenji KAJIWARA \& Marta MAZZOCCO

Generating function associated with the Hankel determinant formula for the solutions of the Painlevé IV equation

MHF2006-15 Raimundas VIDŪNAS

Darboux evaluations of algebraic Gauss hypergeometric functions

MHF2006-16 Masato KIMURA \& Isao WAKANO

New mathematical approach to the energy release rate in crack extension

MHF2006-17 Toru KOMATSU

Arithmetic of the splitting field of Alexander polynomial

MHF2006-18 Hiroki MASUDA

Likelihood estimation of stable Lévy processes from discrete data

MHF2006-19 Hiroshi KAWABI \& Michael RÖCKNER

Essential self-adjointness of Dirichlet operators on a path space with Gibbs measures via an SPDE approach

MHF2006-20 Masahisa TABATA

Energy stable finite element schemes and their applications to two-fluid flow problems

MHF2006-21 Yuzuru INAHAMA \& Hiroshi KAWABI

Asymptotic expansions for the Laplace approximations for Itô functionals of Brownian rough paths

MHF2006-22 Yoshiyuki KAGEI

Resolvent estimates for the linearized compressible Navier-Stokes equation in an infinite layer 
MHF2006-23 Yoshiyuki KAGEI

Asymptotic behavior of the semigroup associated with the linearized

compressible Navier-Stokes equation in an infinite layer

MHF2006-24 Akihiro MIKODA, Shuichi INOKUCHI, Yoshihiro MIZOGUCHI \& Mitsuhiko FUJIO

The number of orbits of box-ball systems

MHF2006-25 Toru FUJII \& Sadanori KONISHI

Multi-class logistic discrimination via wavelet-based functionalization and model selection criteria

MHF2006-26 Taro HAMAMOTO, Kenji KAJIWARA \& Nicholas S. WITTE

Hypergeometric solutions to the $q$-Painlevé equation of type $\left(A_{1}+A_{1}^{\prime}\right)^{(1)}$

MHF2006-27 Hiroshi KAWABI \& Tomohiro MIYOKAWA

The Littlewood-Paley-Stein inequality for diffusion processes on general metric spaces

MHF2006-28 Hiroki MASUDA

Notes on estimating inverse-Gaussian and gamma subordinators under highfrequency sampling

MHF2006-29 Setsuo TANIGUCHI

The heat semigroup and kernel associated with certain non-commutative

harmonic oscillators

MHF2006-30 Setsuo TANIGUCHI

Stochastic analysis and the KdV equation

MHF2006-31 Masato KIMURA, Hideki KOMURA, Masayasu MIMURA, Hidenori MIYOSHI, Takeshi TAKAISHI \& Daishin UEYAMA

Quantitative study of adaptive mesh FEM with localization index of pattern

MHF2007-1 Taro HAMAMOTO \& Kenji KAJIWARA

Hypergeometric solutions to the $q$-Painlevé equation of type $A_{4}^{(1)}$

MHF2007-2 Kouji HASHIMOTO, Kenta KOBAYASHI \& Mitsuhiro T. NAKAO

Verified numerical computation of solutions for the stationary Navier-Stokes equation in nonconvex polygonal domains

MHF2007-3 Kenji KAJIWARA, Marta MAZZOCCO \& Yasuhiro OHTA A remark on the Hankel determinant formula for solutions of the Toda equation

MHF2007-4 Jun-ichi SATO \& Hidefumi KAWASAKI

Discrete fixed point theorems and their application to Nash equilibrium

MHF2007-5 Mitsuhiro T. NAKAO \& Kouji HASHIMOTO

Constructive error estimates of finite element approximations for non-coercive elliptic problems and its applications 
MHF2007-6 Kouji HASHIMOTO

A preconditioned method for saddle point problems

MHF2007-7 Christopher MALON, Seiichi UCHIDA \& Masakazu SUZUKI Mathematical symbol recognition with support vector machines

MHF2007-8 Kenta KOBAYASHI

On the global uniqueness of Stokes' wave of extreme form 